

\title{
Role of Hexadecupole Deformation in the Shape Evolution of Neutron-rich Nd Isotopes
}

\section{Rin Yokoyama*}

Center for Nuclear Study, the University of Tokyo

E-mail: yokoyama@cns.s.u-tokyo.ac.jp

\section{E. Ideguchi}

Research Center for Nuclear Physics, Osaka University

\section{G. Simpson}

LPSC, Grenoble, CNRS/IN2P3

Mn. Tanaka

Research Center for Nuclear Physics, Osaka University

\section{S. Nishimura}

RIKEN Nishina Center

\section{P. Doornenbal}

RIKEN Nishina Center

\section{P.-A. Söderström}

RIKEN Nishina Center

G. Lorusso

RIKEN Nishina Center

Z.Y. Xu

Department of Physics, the University of Tokyo

J. Wu

Department of Physics, Peking University

\section{T. Sumikama}

Department of Physics, Tohoku University

N. Aoi

Research Center for Nuclear Physics, Osaka University

\section{H. Baba}

RIKEN Nishina Center 


\section{F. Bello}

Department of Physics, University of Oslo

\section{F. Browne}

School of Computing Engineering and Mathematics, University of Brighton

\section{R. Daido}

Department of Physics, Osaka University

\section{Y. Fang}

Department of Physics, Osaka University

\section{N. Fukuda}

RIKEN Nishina Center

\section{G. Gey}

ILL, Grenoble

LPSC, Grenoble, CNRS/IN2P3

\section{S. Go}

Center for Nuclear Study, the University of Tokyo

\section{S. Inabe}

RIKEN Nishina Center

\section{T. Isobe}

RIKEN Nishina Center

D. Kameda

RIKEN Nishina Center

\section{K. Kobayashi}

Department of Physics, Rikkyo University

\section{Kobayashi}

Center for Nuclear Study, the University of Tokyo

\section{T. Komatsubara}

Department of Physics, University of Tsukuba

\section{T. Kubo}

RIKEN Nishina Center

\section{Kuti}

MTA Atomki

Z. Li 
Department of Physics, Peking University

\section{Matsushita}

Center for nuclear Study, the University of Tokyo

\section{S. Michimasa}

Center for nuclear Study, the University of Tokyo

\section{C.B. Moon}

Department of Display Engineering, Hoseo University

\section{H. Nishibata}

Department of Physics, Osaka University

\section{Nishizuka}

Department of Physics, Tohoku University

\section{A. Odahara}

Department of Physics, Osaka University

\section{Z. Patel}

Department of Physics, University of Surrey

\section{S. Rice}

Department of Physics, University of Surrey

\section{E. Sahin}

ILL, Grenoble

\section{Sinclair}

Department of Physics, University of York

\section{H. Suzuki}

RIKEN Nishina Center

\section{H. Takeda}

RIKEN Nishina Center

\section{J. Taprogge}

Instituto de Estructura de la Materia, CSIC

Departamento de Fisica Teórica, Universidad Autónoma de Madrid

\section{Zs. Vajta}

MTA Atomki

\section{H. Watanabe}

Department of Physics, Beihang University 


\title{
A. Yagi
}

Department of Physics, Osaka University

\begin{abstract}
A new isomer with $\mu$ s half-life was observed in ${ }^{160} \mathrm{Nd}$ at RIBF, RIKEN Nishina Center by using in-flight fission of a ${ }^{238} \mathrm{U}$ beam and a cluster-type Ge detector array, EURICA. The experimental results and a PSM calculation indicate that the isomer in ${ }^{160} \mathrm{Nd}$ is a 2 quasi-particle excitation of neutrons with a configuration of $v 1 / 2[521] \otimes v 7 / 2[633]$ as the case in other $N=100$ isotones. The $E\left(4^{+}\right) / E\left(2^{+}\right)$ratio of the ground-state band, 3.29, shows the ${ }^{160} \mathrm{Nd}$ is well deformed and the ground band has a rotational nature. The $4^{-}$excitation of ${ }^{160} \mathrm{Nd}$ showed an increase in energy by $\sim 100 \mathrm{keV}$ compared to that of ${ }^{162} \mathrm{Sm}$ as predicted by the PSM calculation. A PSM calculation was performed by changing input $\beta_{4}$ value and confirmed that the large hexadecupole deformation in $\mathrm{Nd}$ was responsible for the increase of the isomer energy.
\end{abstract}

The 26th International Nuclear Physics Conference

11-16 September, 2016

Adelaide, Australia

*Speaker. 


\section{Introduction}

One of the unique features of the nuclear shells is that they have intruder orbitals from larger $N$ quantum number by large spin-orbit interaction. Such shell structure can make a higher-order deformed shape energetically favored in a certain nucleus due to a large interaction between orbitals with higher-order multipolarity.

In stable rare-earth nuclei, a large hexadecupole deformation $\left(\beta_{4}\right)$ is observed in the ground state of $\mathrm{Nd}(Z=60)$ isotopes such as ${ }^{148} \mathrm{Nd}\left(\beta_{4} \sim 0.07\right)$ [1]. Although, in general, quadrupole deformation is sufficient to explain primary phenomena in nuclei, higher-order deformations can cause significant change in the single particle structures. However, higher-order deformations such as hexadecupole deformation have not been well studied for unstable nuclei far from the line of $\beta$ stability. Therefore, it is important to know how such deformation evolves as neutron number increases and how it affects to the single particle levels.

The neutron-rich nuclei in the $A \sim 160$ rare-earth region are known to be well deformed that is evidenced by the $E\left(4^{+}\right) / E\left(2^{+}\right)$ratios of the ground-state rotational bands, $\sim 3.3$ [2]. Many low-lying two quasi particle (qp) isomers with $\sim \mu$ s half-lives with strongly hindered E1 $\gamma$ decays are known in the neutron-rich $\mathrm{Nd}$ and $\mathrm{Sm}$ isotopes [6, 7, 8, 9]. In the even-even $N=100$ isotones from $\mathrm{Sm}$ to $\mathrm{Yb}, 2$-qp isomers with a dominant configuration of $v 1 / 2[521] \otimes v 7 / 2[633]$ are known above $\sim 1 \mathrm{MeV}$ in excitation energy [3,4,5]. It is expected that a 2-qp state with this configuration in ${ }^{160} \mathrm{Nd}$ also can be observed as an isomer. According to the potential energy surface calculation in [9], significantly non zero $\beta_{4}$ and $\beta_{6}$ values are required to reproduce the observed energy of the isomeric state in ${ }^{164} \mathrm{Sm}$. Theoretically, an FRDM [10] and RMF [11] calculations predict large hexadecupole deformation in the neutron-rich $\mathrm{Nd}$ isotopes. It is expected that the large hexadecupole deformation in $\mathrm{Nd}$ isotopes changes the single particle orbitals significantly and it may be observed as the change of excitation energies of isomers.

The experimental results on the $\mathrm{Nd}$ isotopes that will be shown in this article have been published as Ref. [12]. More details of the experimental methods and results can be found in the article.

\section{Experiment}

An isomer spectroscopy study on neutron-rich $Z \sim 60$ isotopes was performed at the RIBF, RIKEN Nishina Center. Neutron-rich $\mathrm{Nd}$ isotopes were produced by using in-flight fission of a $345 \mathrm{MeV} /$ nucleon ${ }^{238} \mathrm{U}$ beam, bombarded on a 4-mm thick Be target. The typical intensity of the ${ }^{238} \mathrm{U}$ beam was $\sim 7 \mathrm{pnA}$. The ions of interest were separated from other fission fragments and identified event-by-event in the BigRIPS, in-flight separator [13]. Particle identification was performed by measuring the time-of-flight (TOF) and magnetic rigidity $(B \rho)$ in the second stage of the BigRIPS and also by the energy loss $(\Delta E)$ in an ion chamber at the final focal plane, F11. The TOF was obtained from the time difference between plastic scintillators at the F3 and F7 achromatic foci. The $B \rho$ value was obtained by the trajectory reconstruction from position and angular information measured by position-sensitive parallel plate avalanche counters (PPACs) [14] at F3, F5, and F7 foci. Details of the procedure for the particle identification at the BigRIPS can be found in [15] 
The measurement was conducted in two different stopper setups at F11. One was optimized for isomer spectroscopy by using a copper passive stopper with 1-mm thick in order to accept a wide range of nuclides with high implantation rates of $\sim 1 \mathrm{kHz}$. In the other setup, an active stopper WAS3ABi [16] consisted of five layers of Double Sided Silicon Strip Detectors (DSSSDs) was used for $\beta-\gamma$ measurements. Each layer of the DSSSDs had $40 \times 60$ strips and each had 1-mm width. In the latter setup with WAS3ABi, the total implantation rate of ions were limited up to $\sim 100 \mathrm{~Hz}$. In total, $1.70 \times 10^{5}$ and $1.34 \times 10^{4}$ ions of ${ }^{158} \mathrm{Nd}$ and ${ }^{160} \mathrm{Nd}$, respectively, were implanted in the two stoppers.

The delayed $\gamma$ rays from the implanted ions were detected by the EURICA [17] array with 12-cluster type HPGe detectors. Each cluster of the EURICA consists of seven crystals. The total detection efficiency of the array for photons of $1332 \mathrm{keV}$ was $\sim 8.4 \%$. The energies of delayed $\gamma$ rays were measured in a time window of $16 \mu$ s following the ion implantation.

\section{Results}



Figure 1: A delayed $\gamma$-ray spectrum for ${ }^{160} \mathrm{Nd}$.

Figure 1 shows a spectrum of delayed $\gamma$ rays detected in coincidence with ${ }^{160} \mathrm{Nd}$ ions. Three peaks at $65.2,149.9$, and $892.8 \mathrm{keV}$ were identified as $\gamma$ rays from the decay of ${ }^{160} \mathrm{Nd}$ isomer. Coincidences was observed between the two $\gamma$ rays at $149.9-$ and $892.8 \mathrm{keV}$, which is an evidence that the two decay form a cascade. Coincidences with 65.2-keV peak were not detected for both of the $\gamma$ rays because the number of peak counts are small for $62.5-\mathrm{keV} \gamma$ ray due to the high internal conversion coefficient. Only the $8.6 \%$ of the $65.2-\mathrm{keV}$ transitions emits $\gamma$ rays according to the internal conversion coefficient for E2 transition given by BrIcc [18]. The 65.2-keV decay is also expected to form a single cascade with other two $\gamma$ rays from the relative intensities. The relative intensities of the three decays including internal conversions were 83(28), 100(7), and 97(8) \% for the 65.2-, 149.9-, and 892.8-keV transitions and agreed with each other. A half-life of 1.63(21) $\mu \mathrm{s}$ was determined for the isomeric states of ${ }^{160} \mathrm{Nd}$ by fitting an exponential function to the time spectrum of ion- $\gamma$ coincidences summed for the three $\gamma$ rays. 


\section{Discussions}

A level scheme was proposed as shown in Fig. 2. The 65.2- and 149.9-keV $\gamma$ rays were assigned as $2^{+} \rightarrow 0^{+}$and $4^{+} \rightarrow 2^{+}$decay of the ground-state rotational band. The energy ratio, $E\left(4^{+}\right) / E\left(2^{+}\right)$, was 3.299 which indicates that the ground-state band of ${ }^{160} \mathrm{Nd}$ nucleus has a rotational nature. The $892.8-\mathrm{keV} \gamma$ ray was assigned to be a decay from isomeric state to the $4^{+}$state of the ground-state band. The isomer is expected to have the same configuration as $4^{-}$isomers in other $N=100$ isotones [3, 4 5], $v 1 / 2[521] \otimes v 7 / 2[633]$. The $B(E 1)$ value for the decay from the isomeric state of ${ }^{160} \mathrm{Nd}$ was $2.0(3) \times 10^{-10} \mathrm{~W}$. u. (Weisskopf units). This value is consistent with the ones from 2-qp isomers in the neighboring isotopes, $9 \times 10^{-9} \geq B(E 1) \geq 3 \times 10^{-12}$,6, , , , 9 .

2-qp states in ${ }^{160} \mathrm{Nd}$ have been predicted by a projected shell model (PSM) calculation [19]. The $4^{-}$state is the lowest 2-qp excitation in the calculation, which is $1.2 \mathrm{MeV}$ above the groundstate. This is very close to our result. The systematics of the $4^{-}$isomers are shown in Fig. 3 with the PSM predictions. The PSM calculation also predicts low-lying $4^{-} 2$-qp state in ${ }^{162} \mathrm{Sm}$. They predict an increase of the isomer energy by $\sim 100 \mathrm{keV}$ from ${ }^{162} \mathrm{Sm}$ to ${ }^{160} \mathrm{Nd}$ by employing larger hexadecupole deformation in $\mathrm{Nd}\left(\beta_{4}=0.032\right)$ than in $\operatorname{Sm}\left(\beta_{4}=0.008\right)$. Our result on ${ }^{160} \mathrm{Nd}$, $1108 \mathrm{keV}$ of the isomer energy also shows a $\sim 100 \mathrm{keV}$ increase from ${ }^{162} \mathrm{Sm}$. In order to identify the effect of the hexadecupole deformation to the single particle energy, we have calculated qp energies of $v 1 / 2[521] \otimes v 7 / 2[633]$ excitation by using a PSM code [20]. By using exactly the same deformation parameters as the calculation in [19], we obtained qp energies of $1.03 \mathrm{MeV}$ for

${ }^{162} \mathrm{Sm}$ and $1.15 \mathrm{MeV}$ for ${ }^{160} \mathrm{Nd}$. As we decreased input $\beta_{4}$ value of ${ }^{160} \mathrm{Nd}$ to 0.008 , the qp energy became lower to $1.01 \mathrm{MeV}$. This result suggests that the increase of qp energy due to the large hexadecupole deformation in $\mathrm{Nd}$ is responsible for the increase of the excitation energy of the $4^{-}$ isomeric state from ${ }^{162} \mathrm{Sm}$ to ${ }^{160} \mathrm{Nd}$.

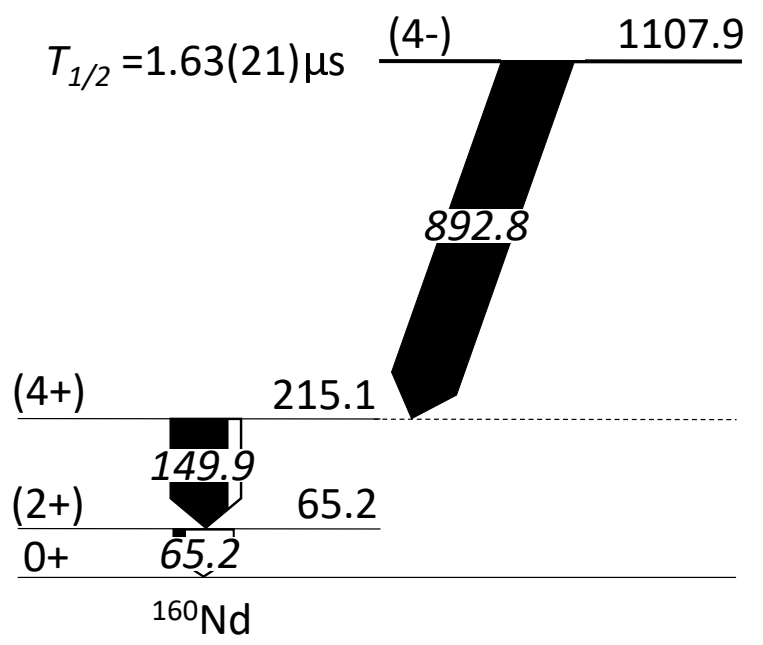

Figure 2: A level scheme of ${ }^{160} \mathrm{Nd}$ proposed from the present experiment. The width of the arrows are proportional to the relative intensity of the corresponding transition. 


\section{过}
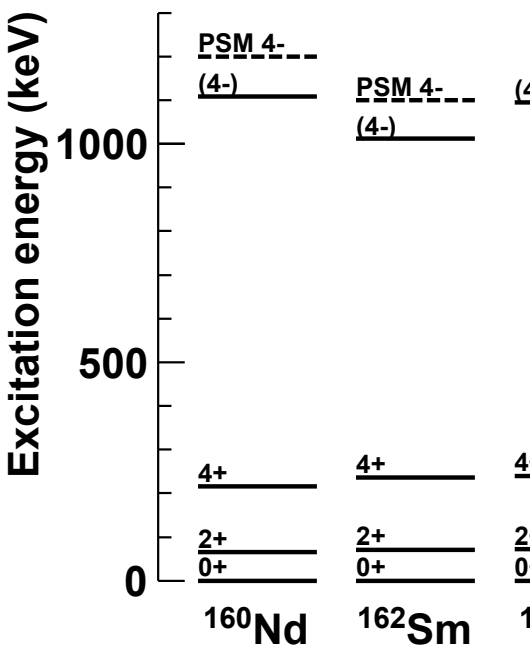

(4-)




Figure 3: Systematics of the excitation energies of $K^{\pi}=4^{-}$isomers and ground-band $4^{+}$and $2^{+}$state. Dashed lines indicates the prediction of PSM calculation [19].

\section{Conclusions}

A new isomer with $\mu$ s half-life was observed in ${ }^{160} \mathrm{Nd}$. The low $E\left(2^{+}\right)$value as $65.2 \mathrm{keV}$ and the $E\left(4^{+}\right) / E\left(2^{+}\right)$ratio of 3.29 indicate the ${ }^{160} \mathrm{Nd}$ nucleus is well deformed and the ground band has a rotational nature. This isomer have been assigned as a 2 qp excitation of neutrons with a configuration of $v 1 / 2[521] \otimes v 7 / 2[633]$ as the case in other $N=100$ isotones. Our result showed an increase of the isomer energy by $\sim 100 \mathrm{keV}$ compared to that of ${ }^{162} \mathrm{Sm}$ as predicted by the PSM calculation. We also confirmed that the large hexadecupole deformation in $\mathrm{Nd}$ was responsible for the increase of the isomer energy in the PSM calculation. This shows that the most neutron-rich $\mathrm{Nd}$ isotopes with its excited states ever measured, ${ }^{160} \mathrm{Nd}$, have large hexadecupole deformation compared to a neighbor nucleus ${ }^{162} \mathrm{Sm}$.

\section{Acknowledgments}

We acknowledge financial support from KAKENHI (25247045). This work was carried out at the RIBF operated by RIKEN Nishina Center, RIKEN and CNS, the University of Tokyo. We acknowledge the EUROBALL Owners Committee for the loan of germanium detectors and the PreSpec Collaboration for the readout electronics of the cluster detectors. Part of the WAS3ABi was supported by the Rare Isotope Science Project which is funded by the Ministry of Science, ICT and Future Planning (MSIP) and National Research Foundation (NRF) of Korea. This work was supported by the Spanish Ministerio de Ciencia e Innovación under Contracts No. FPA2009-13377C02 and FPA2011-29854-C04 and the Hungarian Research Fund (OTKA), Contract No. K100835. The author R.Y. was supported by ALPS program by the University of Tokyo and Grant-in-aid for JSPS fellow. 


\section{References}

[1] B. S. N. Singh et al., J. Physics 61, 507 (2003)

[2] B. Pritychenko et al., At. Data Nucl. Data Tables 107, 1 (2016)

[3] P. M. Walker et al., Nucl. Phys. A 365, 61 (1998)

[4] C. Y. Wu et al., Phys. Rev. C 68, 044305 (2003)

[5] S. Go et al., RIKEN Accel. Prog. Rep. 46, 21 (2013)

[6] C. Gautherin et al., Eur. Phys. J. A 1, 391 (1998)

[7] G. S. Simpson et al., Phys. Rev. C 80, 024304 (2009)

[8] E. Yeoh et al., Eur. Phys. J. A 45, 147 (2010)

[9] Z. Patel et al., Phys. Rev. Lett. 113, 262502 (2014)

[10] P. Möller et al., At. Data Nucl. Data Tables 59, 185 (1995)

[11] G. Lalazissis et al., At. Data Nucl. Data Tables 71, 1 (1999)

[12] E. Ideguchi et al., Phys. Rev. C 94, 064322 (2016)

[13] T. Kubo et al., NIM B 204, 97 (2003)

[14] H. Kumagai et al., NIM A 470, 562 (2001)

[15] T. Ohnishi et al., J. Phys. Soc. Jpn. 79, 073201 (2010)

[16] S. Nishimura et al., Nucl. Phys. News 22, No.3 (2012)

[17] S. Nishimura et al., RIKEN Accel. Prog. Rep. 46, 182 (2013)

[18] T. Kibédi et al., NIM A, 589, 202 (2008)

[19] Y. C. Yang et al., J. Phys. G. Nucl. Part. Phys. 37, 085110 (2010)

[20] Y. Sun et al., Comput. Phys. Commun. 104, 245 (1997) 